\title{
SIMPLICITY OF THE BERGMAN, SZEGÖ AND POISSON KERNEL FUNCTIONS
}

\author{
SteVen R. BeLL
}

\begin{abstract}
A вstract. We announce a proof that the Bergman, Szegö, and Poisson kernels associated to a finitely connected domain in the plane are simple in the sense that they are not genuine functions of two variables. They are all composed of finitely many holomorphic functions of one variable. We can also prove that the kernels cannot be too simple by showing that the only finitely connected domains in the plane whose Bergman or Poisson kernels are rational functions are the simply connected domains which can be mapped onto the unit disc by a rational biholomorphic mapping. This leads to a proof that the classical Green's function associated to a finitely connected domain in the plane is one half the logarithm of a real-valued rational function if and only if the domain is simply connected and there is a rational biholomorphic map of the domain onto the unit disc.
\end{abstract}

\section{Introduction}

There are many places in the study of functions of one complex variable where functions of two complex variables arise, and it is in one of these places that I, a hungry analyst of functions of several complex variables, have recently gone to graze. This paper reports on how I have found the quality of the fodder to be wholesome, but disappointing from the viewpoint of several complex variables.

The Bergman and Szegö kernels associated to a bounded domain in the plane are functions of two complex variables that carry encoded within them a great deal of information about the domain. Conformal mappings onto canonical domains, classical domain functions, and other important objects of potential theory can be expressed simply in terms of the Bergman and Szegö kernels. It is therefore widely believed that these kernels are highly transcendental and difficult to compute. The purpose of this paper

1991 Mathematics Subject Classification. 32H10.

Key words and phrases. Bergman kernel, Szegő kernel, Green's function, Poisson kernel.

Received November 21, 1994.

Research supported by NSF grant DMS-9302513. 
is to announce results that show that the kernel functions are not nearly as complex as one might expect. They are not genuine functions of two complex variables. Rather, they are simple rational combinations of finitely many functions of one complex variable. Full details of the proofs of the results will appear in [4] and [5].

It has long been known that the kernel functions associated to a simply connected domain are no more complex than a single Riemann mapping function. Indeed, let $a$ be a fixed point in a simply connected domain $\Omega \neq \mathbb{C}$ and let $f_{a}(z)$ denote the Riemann mapping function mapping $\Omega$ one-to-one onto the unit disc $D_{1}(0)$ with $f_{a}(a)=0$ and $f_{a}^{\prime}(a)>0$. Let $S(z, w)$ denote the Szegö kernel associated to $\Omega$. The Riemann map $f_{a}$ may be expressed simply in terms of $S(z, a)$ and $S(z, w)$ is given by

$$
S(z, w)=\frac{c S(z, a) \overline{S(w, a)}}{1-f_{a}(z) \overline{f_{a}(w)}},
$$

where $c=1 / S(a, a)$. A similar identity holds for the Bergman kernel,

$$
K(z, w)=\frac{4 \pi c^{2} S(z, a)^{2} \overline{S(w, a)^{2}}}{\left(1-f_{a}(z) \overline{f_{a}(w)}\right)^{2}}
$$

where $c=1 / S(a, a)$. This shows that the Bergman kernel is composed of the same basic functions that make up the Szegö kernel. Finally, the Poisson kernel $p(z, w)$ is given by

$$
p(z, w)=\frac{S(z, w) S(w, a)}{S(z, a)}+\frac{\overline{S(z, w) S(w, a) f_{a}(z)}}{\overline{S(z, a) f_{a}(w)}}
$$

where $z$ is a point in $\Omega$ and $w$ is a point in the boundary (see [1, page 37]). Thus, the Poisson kernel is also composed of the same basic functions. These formulas for the kernel functions in a simply connected domain are not new. However, we prove analogous results for $n$-connected domains that are new. The new results show that there are $n+1$ basic functions that comprise all the kernels. An interesting feature of the results in this paper is the central role played by the Ahlfors map and by the zeroes of the Szegö kernel.

We can prove our main results about the kernel functions in general finitely connected domains such that no boundary component reduces to a point. In case $\Omega$ is a bounded finitely connected domain in the plane with $C^{\infty}$ smooth boundary, our results reveal that all the kernel functions are composed of basic holomorphic functions that are all given as solutions 
to explicit Kerzman-Stein integral equations. All elements of the kernel functions may be computed by means of simple linear algebra and one dimensional integrals and one dimensional integral equations. At no point is a double integral with respect to area measure needed. This result is particularly surprising for the Bergman kernel function associated to a multiply connected domain. A traditional way to compute the Bergman kernel of such a domain has been to orthonormalize a set of rational functions that span a dense subset of the Bergman space. This is a numerical nightmare compared to the methods we establish in [5].

In the last section of this paper, we describe results from [4] that give conditions on a domain for its Bergman, Szegő, or Poisson kernel function to be a rational function. We prove in [4], for example, that the Bergman kernel associated to a finitely connected domain is rational exactly when the domain is simply connected and is biholomorphic to the unit disc via a rational mapping. This result about the Bergman kernel has as a corollary that the Green's function associated to a finitely connected domain is one half the logarithm of a rational function if and only if the domain is simply connected and there is a rational biholomorphic map of the domain onto the unit disc.

\section{Preliminaries}

Before we can state our main theorems, we must review some basic facts about the Szegö kernel and the Ahlfors mapping. (Proofs of these facts can be found in [6] and [1].)

Suppose that $\Omega$ is a bounded $n$-connected domain in the plane with $C^{\infty}$ smooth boundary. Let $\gamma_{j}, j=1, \ldots, n$, denote the $n$ non-intersecting $C^{\infty}$ simple closed curves which define the boundary of $\Omega$, and suppose that $\gamma_{j}$ is parameterized in the standard sense by $z_{j}(t), 0 \leq t \leq 1$. Let $T(z)$ be the $C^{\infty}$ function defined on $b \Omega$ such that $T(z)$ is the complex number representing the unit tangent vector at $z \in b \Omega$ pointing in the direction of the standard orientation. This complex unit tangent vector function is characterized by the equation $T\left(z_{j}(t)\right)=z_{j}^{\prime}(t) /\left|z_{j}^{\prime}(t)\right|$.

We shall let $A^{\infty}(\Omega)$ denote the space of holomorphic functions on $\Omega$ that are in $C^{\infty}(\bar{\Omega})$. The space of complex valued functions on $\Omega$ that are square integrable with respect to Lebesgue area measure $d A$ will be written $L^{2}(\Omega)$, and the space of complex valued functions on $b \Omega$ that are square integrable with respect to arc length measure $d s$ will be denoted by $L^{2}(b \Omega)$. The Bergman space of holomorphic functions on $\Omega$ that are in $L^{2}(\Omega)$ shall be written $H^{2}(\Omega)$ and the Hardy space of functions in $L^{2}(b \Omega)$ that are the $L^{2}$ boundary values of holomorphic functions on $\Omega$ shall be written $H^{2}(b \Omega)$. Let $\langle u, v\rangle_{b \Omega}$ denote the inner product on $L^{2}(b \Omega)$ and $\langle u, v\rangle_{\Omega}$ denote the 
inner product on $L^{2}(\Omega)$.

For each fixed point $a \in \Omega$, the Szegö kernel $S(z, a)$, as a function of $z$, extends to the boundary to be a function in $A^{\infty}(\Omega)$. Furthermore, $S(z, a)$ has exactly $(n-1)$ zeroes in $\Omega$ (counting multiplicities) and does not vanish at any points $z$ in the boundary of $\Omega$. The Garabedian kernel $L(z, a)$ is a kernel related to the Szegö kernel via the identity

$$
\frac{1}{i} L(z, a) T(z)=S(a, z) \quad \text { for } z \in b \Omega \text { and } a \in \Omega .
$$

For fixed $a \in \Omega$, the kernel $L(z, a)$ is a holomorphic function of $z$ on $\Omega-\{a\}$ with a simple pole at $a$ with residue $1 /(2 \pi)$. Furthermore, as a function of $z, L(z, a)$ extends to the boundary and is in the space $C^{\infty}(\bar{\Omega}-\{a\})$. In fact, $L(z, a)$ extends to be in $C^{\infty}((\bar{\Omega} \times \bar{\Omega})-\{(z, z): z \in \bar{\Omega}\})$. Also, $L(z, a)$ is nonzero for all $(z, a)$ in $\bar{\Omega} \times \Omega$ with $z \neq a$.

The kernel $S(z, w)$ is holomorphic in $z$ and antiholomorphic in $w$ on $\Omega \times \Omega$, and $L(z, w)$ is holomorphic in both variables for $z, w \in \Omega, z \neq w$. We note that $S(z, z)$ is real and positive for each $z \in \Omega$, and that $S(z, w)=$ $\overline{S(w, z)}$ and $L(z, w)=-L(w, z)$. The Szegö kernel reproduces holomorphic functions in the sense that

$$
h(a)=\langle h, S(\cdot, a)\rangle_{b \Omega}
$$

for all $h \in H^{2}(b \Omega)$ and $a \in \Omega$.

Given a point $a \in \Omega$, the Ahlfors map $f_{a}$ associated to the pair $(\Omega, a)$ is a proper holomorphic mapping of $\Omega$ onto the unit disc. It is an $n$-toone mapping (counting multiplicities), it extends to be in $A^{\infty}(\Omega)$, and it maps each boundary curve $\gamma_{j}$ one-to-one onto the unit circle. Furthermore, $f_{a}(a)=0$, and $f_{a}$ is the unique function mapping $\Omega$ into the unit disc maximizing the quantity $\left|f_{a}^{\prime}(a)\right|$ with $f_{a}^{\prime}(a)>0$. The Ahlfors map is related to the Szegő kernel and Garabedian kernel via

$$
f_{a}(z)=\frac{S(z, a)}{L(z, a)} .
$$

Also, $f_{a}^{\prime}(a)=2 \pi S(a, a) \neq 0$. Because $f_{a}$ is $n$-to-one, $f_{a}$ has $n$ zeroes. The simple pole of $L(z, a)$ at $a$ accounts for the simple zero of $f_{a}$ at $a$. The other $n-1$ zeroes of $f_{a}$ are given by $(n-1)$ zeroes of $S(z, a)$ in $\Omega-\{a\}$. Let $a_{1}, a_{2}, \ldots, a_{n-1}$ denote these $n-1$ zeroes (counted with multiplicity). I proved in [3] (see also [1, page 105]) that, if $a$ is close to one of the boundary curves, the zeroes $a_{1}, \ldots, a_{n-1}$ become distinct simple zeroes. It follows from this result that, for all but at most finitely many points $a \in \Omega$, $S(z, a)$ has $n-1$ distinct simple zeroes in $\Omega$ as a function of $z$. 


\section{A formula for the Szegö kernel}

The zeroes of the Szegö kernel give rise to a particularly nice basis for the Hardy space of an $n$-connected domain with $C^{\infty}$ smooth boundary, and this basis allows us to write down a formula for the Szegö kernel that reveals its complexity. We shall use the notation that we set up in the preceding section. We assume that $a \in \Omega$ is a fixed point in $\Omega$ that has been chosen so that the $n-1$ zeroes, $a_{1}, \ldots, a_{n-1}$, of $S(z, a)$ are distinct and simple.

Theorem 3.1. The Szegö kernel is given by

$$
S(z, w)=\frac{1}{1-f(z) \overline{f(w)}}\left(c_{0} S(z, a) \overline{S(w, a)}+\sum_{i, j=1}^{n-1} c_{i j} S\left(z, a_{i}\right) \overline{S\left(w, a_{j}\right)}\right)
$$

where $f(z)$ denotes the Ahlfors map $f_{a}(z), c_{0}=1 / S(a, a)$, and the coefficients $c_{i j}$ are given as the coefficients of the inverse matrix to the matrix $\left[S\left(a_{j}, a_{k}\right)\right]$.

We now sketch the proof of Theorem 3.1. The proof uses a special basis for the Hardy space. For convenience, let $a_{0}$ denote $a$. It is shown in [4] that the set of functions

$$
h_{i k}(z)=S\left(z, a_{i}\right) f(z)^{k}
$$

$0 \leq i \leq n-1$, and $k \geq 0$, forms a basis for the Hardy space $H^{2}(b \Omega)$. Furthermore,

$$
\left\langle h_{i k}, h_{j m}\right\rangle_{b \Omega}= \begin{cases}0, & \text { if } k \neq m \\ S\left(a_{j}, a_{i}\right), & \text { if } k=m .\end{cases}
$$

Orthonormalize the basis via the Gram-Schmidt procedure. Identity (3.1) shows that most of the functions in the sequence are already orthogonal. We need only fix $k$ and orthonormalize the $n$ functions $S\left(z, a_{i}\right) f(z)^{k}$, $i=0,1, \ldots, n-1$. We obtain an orthonormal set $\left\{H_{i k}\right\}$ given by

$$
\begin{gathered}
H_{0 k}(z)=b_{00} S(z, a) f(z)^{k} \quad \text { and } \\
H_{i k}(z)=\sum_{j=1}^{i} b_{i j} S\left(z, a_{j}\right) f(z)^{k}, \quad i=1, \ldots, n-1,
\end{gathered}
$$

where $b_{i i} \neq 0$ for each $i=0,1, \ldots, n-1$. Because $|f|=1$ on $b \Omega$, it follows that the coefficients $b_{i j}$ do not depend on $k$. 
The Szegö kernel can be written in terms of our orthonormal basis as

$$
S(z, w)=\sum_{i=0}^{n-1} \sum_{k=0}^{\infty} H_{i k}(z) \overline{H_{i k}(w)}
$$

The sum

$$
\sum_{k=0}^{\infty} f(z)^{k} \overline{f(w)^{k}}=\frac{1}{1-f(z) \overline{f(w)}}
$$

can be factored from the expression for $S(z, w)$ to yield the formula in the statement of Theorem 3.1. To see that $c_{0}=1 / S(a, a)$, set $z=a$ and $w=a$ in the formula. The values of the other coefficients can be deduced by setting $w=a_{k}, k=1, \ldots, n-1$ in the formula and by noting that $f\left(a_{k}\right)=0$ and $S\left(a, a_{k}\right)=0$. The details may be found in [4].

\section{The Bergman kernel}

In this section, we shall see that the Bergman kernel of an $n$-connected domain in the plane with $C^{\infty}$ smooth boundary is composed of the same basic functions that comprise the Szegö kernel.

The Bergman kernel $K(z, w)$ is related to the Szegő kernel via the identity

$$
K(z, w)=4 \pi S(z, w)^{2}+\sum_{i, j=1}^{n-1} A_{i j} F_{i}^{\prime}(z) \overline{F_{j}^{\prime}(w)},
$$

where the functions $F_{i}^{\prime}(z)$ are classical functions of potential theory described as follows. The harmonic measure function $\omega_{j}$ which solves the Dirichlet problem on $\Omega$ with boundary data equal to one on the boundary curve $\gamma_{j}$ and zero on $\gamma_{k}$ if $k \neq j$ has a multivalued harmonic conjugate. The function $F_{j}^{\prime}(z)$ is a globally defined single valued holomorphic function on $\Omega$ which is locally defined as the derivative of $\omega_{j}+i v$ where $v$ is a local harmonic conjugate for $\omega_{j}$. The Cauchy-Riemann equations reveal that $F_{j}^{\prime}(z)=2\left(\partial \omega_{j} / \partial z\right)$.

Let $\mathcal{F}^{\prime}$ denote the complex linear span of the set of functions $\left\{F_{j}^{\prime}(z)\right.$ : $j=1, \ldots, n-1\}$. It is a classical fact that $\mathcal{F}^{\prime}$ is $n-1$ dimensional. Notice that $S\left(z, a_{i}\right) L(z, a)$ is in $A^{\infty}(\Omega)$ because the pole of $L(z, a)$ at $z=a$ is cancelled by the zero of $S\left(z, a_{i}\right)$ at $z=a$. Schiffer proved (see $\left.[9,1,3]\right)$ that the $n-1$ functions $S\left(z, a_{i}\right) L(z, a), i=1, \ldots, n-1$ form a basis for $\mathcal{F}^{\prime}$. We may now write

$$
K(z, w)=4 \pi S(z, w)^{2}+\sum_{i, j=1}^{n-1} \lambda_{i j} S\left(z, a_{i}\right) L(z, a) \overline{S\left(w, a_{j}\right) L(w, a)},
$$


which, together with Theorem 3.1, shows that the Bergman kernel is no more complex than the Szegö kernel.

Theorem 4.1. The Bergman kernel is a rational combination of the same finitely many basic functions that make up the Szegö kernel.

Formula (4.1) describes the Bergman kernel in a domain with smooth boundary. If a finitely connected domain does not have smooth boundary, and if none of its boundary components are points, it can be mapped conformally onto a domain whose boundary is smooth. The transformation formula for the Bergman kernels under biholomorphic mappings can then be used to obtain the following theorem.

Theorem 4.2. Suppose $\Omega$ is a finitely connected domain such that no boundary component of $\Omega$ is a point. Let $f(z)$ denote an Ahlfors map of $\Omega$ onto the unit disc. The Bergman kernel $K(z, w)$ associated to $\Omega$ is a function of the form

$$
\begin{aligned}
K(z, w)=\frac{1}{(1-f(z) \overline{f(w)})^{2}}\left(\sum_{j, k=1}^{n(n+1) / 2} C_{j k} H_{j}(z) \overline{H_{k}(w)}\right) & \\
& +\sum_{i, j=1}^{n-1} \lambda_{i j} G_{i}(z) \overline{G_{j}(w)}
\end{aligned}
$$

where the functions $H_{j}$ and $G_{j}$ are functions of one variable in the Bergman space.

\section{Complexity of the Poisson kernel}

I showed in [3] how the Szegö projection can be used to solve the Dirichlet problem. The method gives rise to a formula for the Poisson kernel in terms of the Szegö kernel. Let $\Omega$ be a bounded $n$-connected domain with $C^{\infty}$ smooth boundary. As above, we select a point $a \in \Omega$ such that the zeroes $a_{1}, \ldots, a_{n-1}$ of $S(z, a)$ are all distinct and simple. Let $S_{a}(z)=S(z, a)$ and 
$L_{a}(z)=L(z, a)$. The Poisson kernel $p(z, w)$ is given by

$$
\begin{gathered}
p(z, w)=\frac{S(z, w) S(w, a)}{S(z, a)}+\frac{\overline{S(z, w) L(w, a)}}{\overline{L(z, a)}} \\
-\sum_{j, k=1}^{n-1}\left(B_{j k} S\left(a_{k}, w\right) S(w, a) \int_{\zeta \in \gamma_{j}} \frac{S(z, \zeta) S(\zeta, a)}{S(z, a)} d s\right) \\
-\sum_{j, k=1}^{n-1}\left(\overline{B_{j k} S\left(a_{k}, w\right) S(w, a)} \int_{\zeta \in \gamma_{j}} \frac{\overline{S(z, \zeta) L(\zeta, a)}}{\overline{L(z, a)}} d s\right) \\
+\sum_{j=1}^{n-1} \omega_{j}(z)\left(\sum_{k=1}^{n-1} B_{j k} S\left(a_{k}, w\right) S(w, a)\right)
\end{gathered}
$$

where $B_{j k}$ are constants whose explicit values are given in [4].

The first term in the sum on the right hand side of (5.1) is a meromorphic function in $z$ with simple poles at the zeroes of $S(z, a)$. The second term is antiholomorphic in $z$ with no poles. The third term is meromorphic in $z$ with simple poles at the zeroes of $S(z, a)$ that exactly cancel the simple poles of the first term. The fourth term is antiholomorphic in $z$ with no poles. The last term is harmonic in $z$. This formula, although rather ugly, is built up of functions of one complex variable.

We show in [4] that formula (5.1) can be manipulated to yield the shorter identity

$$
p(a, w)=\frac{|S(w, a)|^{2}}{S(a, a)}+\sum_{j=1}^{n-1}\left(\omega_{j}(a)-\lambda_{j}(a)\right) \mu_{j}(w)
$$

where the $\lambda_{j}$ are non-harmonic functions in $C^{\infty}(\bar{\Omega})$ that have the same boundary behavior as the harmonic measure functions and the $\mu_{j}$ are $C^{\infty}$ functions on $b \Omega$. The $\mu_{j}$ can be expressed directly in terms of the Szego" and Garabedian kernels and the $\lambda_{j}$ can be written explicitly in terms of simple integrals of the Szegö kernel. This last formula relates the Poisson kernel $p(z, w)$ to the Poisson-Szegö kernel $|S(w, a)|^{2} / S(a, a)$ in a multiply connected domain. (These two kernels are equal in simply connected domains.)

Formula (5.1) can be used to write the Poisson kernel as

$$
\begin{aligned}
p(z, w)= & \operatorname{Re}\left[\frac{\sum_{i, j=1}^{n} h_{i}(z) \overline{H_{j}(w) T(w)}}{1-f(z) \overline{f(w)}}+\sum_{j=1}^{n-1} A_{j}(z) \overline{B_{j}(w) T(w)}\right] \\
& +h_{0}(w)+\overline{H_{0}(w) T(w)}+\sum_{j=1}^{n-1} \omega_{j}(z) \overline{g_{j}(w) T(w)}
\end{aligned}
$$


where $f(z)$ denotes an Ahlfors mapping of $\Omega$ onto the unit disc, and the other functions, $h_{j}, H_{j}, A_{j}, B_{j}$, and $g_{j}$, are all holomorphic functions of one variable in $A^{\infty}(\Omega)$. Recall that Ahlfors maps are also in $A^{\infty}(\Omega)$. Hence, the Poisson kernel is formed by taking simple combinations of the functions $\omega_{j}$ and finitely many holomorphic functions in $A^{\infty}(\Omega)$ of one variable.

\section{The Green's function}

Because the Poisson kernel is related to the gradient of the classical Green's function, the results of the previous section can be used to deduce that the gradient of the Green's function is composed of finitely many functions of one variable that are all in $C^{\infty}(\bar{\Omega})$. Indeed, (2.1) and (5.1) can be used to show that

$$
p(z, w)=i \frac{S(z, w) \overline{L(w, z) T(w)}}{S(z, z)}+\sum_{j=1}^{n-1}\left(\omega_{j}(z)-\lambda_{j}(z)\right) \overline{g_{j}(w) T(w)}
$$

where $g_{j}(w)=-i \sum_{k=1}^{n-1} \overline{B_{j k}} S\left(w, a_{k}\right) L(w, a)$, and since

$$
p(z, w)=\frac{1}{2 \pi} \frac{\partial}{\partial n_{w}} G(z, w)=\frac{i}{\pi} \frac{\partial}{\partial \bar{w}} G(z, w) \overline{T(w)},
$$

we may equate these two expressions. The resulting identity extends to hold for all $w$ in $\Omega$.

Theorem 6.1. Suppose that $\Omega$ is a bounded $n$-connected domain with $C^{\infty}$ smooth boundary. The Green's function associated to $\Omega$ satisfies

$$
\frac{\partial G}{\partial \bar{w}}(z, w)=\pi\left(\frac{S(z, w) \overline{L(w, z)}}{S(z, z)}-i \sum_{j=1}^{n-1}\left(\omega_{j}(z)-\lambda_{j}(z)\right) \overline{g_{j}(w)}\right)
$$

for all $z, w \in \Omega, z \neq w$.

Theorem 3.1 asserts that the Szegö kernel is composed of finitely many functions of one variable, and since $L(z, w)$ satisfies an analogous identity (see [4]), it follows from Theorem 6.1 that the gradient of the Green's function is also composed of finitely many functions of one variable in $C^{\infty}(\bar{\Omega})$.

\section{Characterization of domains with rational kernel functions}

In the previous sections, we have explained why the kernel functions are not as complex as one might expect them to be. In this section, we shall 
describe theorems that say that the only domains whose kernel functions are rational are the simply connected domains given as $\{z:|F(z)|<1\}$ for some rational function $F(z)$.

A function $R(z, w)$ of two complex variables is called rational if there are relatively prime polynomials $P(z, w)$ and $Q(z, w)$ such that $R(z, w)=$ $P(z, w) / Q(z, w)$. It is not hard to prove that a function $H(z, w)$ which is holomorphic in $z$ and $w$ on a product domain $\Omega_{1} \times \Omega_{2}$ is rational if and only if for each fixed $b \in \Omega_{2}$, the function $H(z, b)$ is rational in $z$, and for each fixed $a \in \Omega_{1}$, the function $H(a, w)$ is rational in $w$ (see Bochner and Martin [7, page 201]). We shall say that the Bergman kernel function $K(z, w)$ associated to a domain $\Omega$ is rational if it can be written as $R(z, \bar{w})$ where $R$ is a holomorphic rational function of two variables. Because the Bergman kernel is hermitian, the facts above imply that $K(z, w)$ is rational if and only if for each point $a \in \Omega$, the function $K(z, a)$ is a rational function of $z$. In fact, $K(z, w)$ is rational if and only if there exists a small disc $D_{\epsilon}\left(w_{0}\right) \subset \Omega$ such that $K(z, a)$ is a rational function of $z$ for each $a \in D_{\epsilon}\left(w_{0}\right)$. Similar statements hold for the other kernel functions.

Theorem 7.1. Suppose $\Omega$ is a bounded n-connected domain, $n>1$, with $C^{\infty}$ smooth boundary. Neither the Bergman kernel nor the Szegö kernel associated to $\Omega$ can be rational functions.

The assumption in Theorem 7.1 that the boundary of $\Omega$ is $C^{\infty}$ smooth can be relaxed. For example, the conclusion about the Bergman kernel holds if the domain is only assumed to be finitely connected and such that no boundary component is a point. We explain in [4] how to relax the smoothness assumptions.

Theorem 7.1 together with the following theorem characterize those domains in the plane with rational Bergman or Szegö kernels.

Theorem 7.2. Suppose $\Omega \neq \mathbb{C}$ is a simply connected domain; the Bergman kernel associated to $\Omega$ is rational if and only if there is a rational biholomorphic mapping $f(z)$ mapping $\Omega$ one-to-one onto the unit disc. The Szego" kernel associated to $\Omega$ is rational if and only if there is a rational biholomorphic mapping $f(z)$ mapping $\Omega$ one-to-one onto the unit disc such that $f^{\prime}(z)$ is the square of a rational function.

Since the Bergman kernel is equal to a second partial derivative of the Green's function, Theorem 7.1 and 7.2 yield the following theorem.

Theorem 7.3. Suppose $\Omega$ is a finitely connected domain such that no boundary component of $\Omega$ is a point. The Green's function $G(z, a)$ associated to $\Omega$ is $1 / 2$ the logarithm of a real-valued rational function of the four real variables given by the real and imaginary parts of $z$ and $a$ if and only 
if $\Omega$ is simply connected and there is a rational biholomorphic mapping of $\Omega$ onto the unit disc. Furthermore, the Bergman kernel $K(z, w)$ associated to $\Omega$ is rational if and only if $\Omega$ is simply connected and equivalent to the disc via a rational biholomorphic mapping.

Hence, the only finitely connected domains having Green's functions as simple as the Green's function for the disc are the obvious ones. (Of course, the Green's function itself can never be rational because it has a logarithmic singularity.)

Finally, we mention that similar results hold for the Poisson kernel.

Theorem 7.4. Suppose that $\Omega$ is a bounded $n$-connected domain with $C^{\infty}$ smooth boundary. The Poisson kernel $p(z, w)$ associated to $\Omega$ is such that $p(z, w)$ is a rational function of the real and imaginary parts of $z \in \Omega$ for $w$ in an open subset of the boundary if and only if $n=1$ and $\Omega$ is biholomorphic to the unit disc via a rational map $f: \Omega \rightarrow D_{1}(0)$.

\section{References}

1. S. Bell, The Cauchy transform, potential theory, and conformal mapping, CRC Press, Boca Raton, 1992.

2. Numerical computation of the Ahlfors map of a multiply connected planar domain, J. Math. Anal. and Appl. 120 (1986), 211-217.

3. - The Szegö projection and the classical objects of potential theory in the plane, Duke Math. J. 64 (1991), 1-26.

4. Complexity of the classical kernel functions of potential theory, to appear.

5. __ A recipe for the Bergman kernel function of a multiply connected domain, to appear.

6. S. Bergman, The kernel function and conformal mapping, Math. Surveys 5, AMS, Providence, 1950.

7. S. Bochner and W. Martin, Several complex variables, Princeton Univ. Press, Princeton, 1948.

8. N. Kerzman and E. M. Stein, The Cauchy kernel, the Szegö kernel, and the Riemann mapping function, Math. Ann. 236 (1978), 85-93.

9. M. Schiffer, Various types of orthogonalization, Duke Math. J. 17 (1950), 329-366.

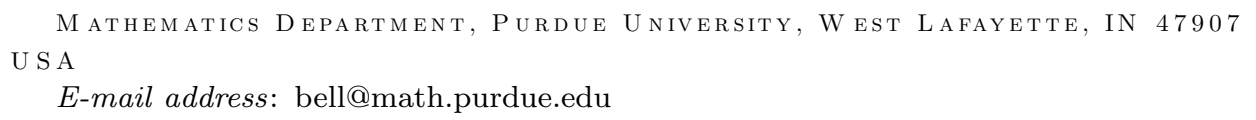

